\title{
The Transmittance Estimation of Two Layers Coating TP Film by Neural Network
}

\author{
Du-Jou Huang ${ }^{1}$, Yu-Ju Chen ${ }^{2}$, Chuo-Yean Chang ${ }^{3}$, Huang-Chu Huang ${ }^{4}$ and Rey-Chue Hwang ${ }^{1, *}$ \\ ${ }^{1}$ Department of Electrical Engineering, I-Shou University, Kaohsiung, Taiwan \\ 2 Department of Information Management, Cheng Shiu University, Kaohsiung, Taiwan \\ ${ }^{3}$ Department of Electrical Engineering, Cheng Shiu University, Kaohsiung, Taiwan \\ ${ }^{4}$ Department of Electric Communication, National Kaohsiung Marine University, Kaohsiung, Taiwan
}

Received: 14 Jun. 2013, Revised: 20 Oct. 2013, Accepted: 22 Oct. 2013

Published online: 1 Jan. 2014

\begin{abstract}
In this paper, the estimations for the optical property of touch panel (TP) decoration film with two layers coating are presented. The technique of neural network is used to develop an artificial intelligent (AI) TP transmittance estimator which is able to catch the complicated relationship between TP transmittance and its all possible influencing factors, such as the compositions of coating material, the thickness of coating, the rotation speed of evaporator?s holder and so on. This AI estimator then can provide the useful information which could help the engineer to set the relevant control parameters of evaporator properly before the evaporation process is taken. The simulation results show that such an AI system is quite promising to be developed.
\end{abstract}

Keywords: Estimation, Touch panel, Transmittance, Neural Network.

\section{Introduction}

In recent years, TP has become an indispensable part for many electronic products, such as the tablet computer, smart phone, digital camera and ticket vendor. According to the trend of science and technology development, TP will certainly be used in the smart life environment of human beings more and more in the future. Basically, the film coating is an essential work in the manufacturing process of $\mathrm{TP}$ and the common equipment used for coating process is vacuum evaporator. The diagram of touch panel structure is shown in Fig. 1. [1,2,3,4,5,6].

As mentioned above, the coating process of TP film is usually processed by the vacuum evaporator which is used in a wide range of industrial applications. However, before the evaporation process is started, the relevant control parameters of evaporator must be set properly by the engineer with full experience. Besides, many variables such as the composition of coating target, the coating layer, the thickness of coating layer, the speed of evaporation, are all the important influencing factors that might determine the quality of TP coating film [5,6]. Usually, the transmittance and chromatic aberration (L.a.b. values) are two optical properties used for judging the quality of TP film. Two properties are highly correlated with the influencing factors above. The relationship between film?s optical properties and influencing factors is very complicated. It is hardly be modeled by a certain mathematic function. In most of evaporation processes, the setting of control parameters of evaporator is still based on the trial-and-error method. Thus, how to find a solution for setting the proper control parameters of evaporator is the aim of this research.

In last two decades, neural network (NN) has been widely applied into the various science and management areas due to its powerful learning and mapping capabilities $[5,6,7,8,9,10,11,12]$. The complex relationship between input and output pairs of the training data could be easily developed through a simple training process. Thus, NN technique has been concluded as one of the best tools to solve the nonlinear mapping problem. It has found a huge variety of uses in various applications.

In this research, the estimation for the optical property of TP film with two layers coating is considered. The neural network is the main tool used for obtaining the relationship between TP film?s transmittance and all possible influencing factors. The TP film has two layers coating. One coating target is $\mathrm{Cr}$ and the other one

\footnotetext{
* Corresponding author e-mail: rchwang@ isu.edu.tw
} 


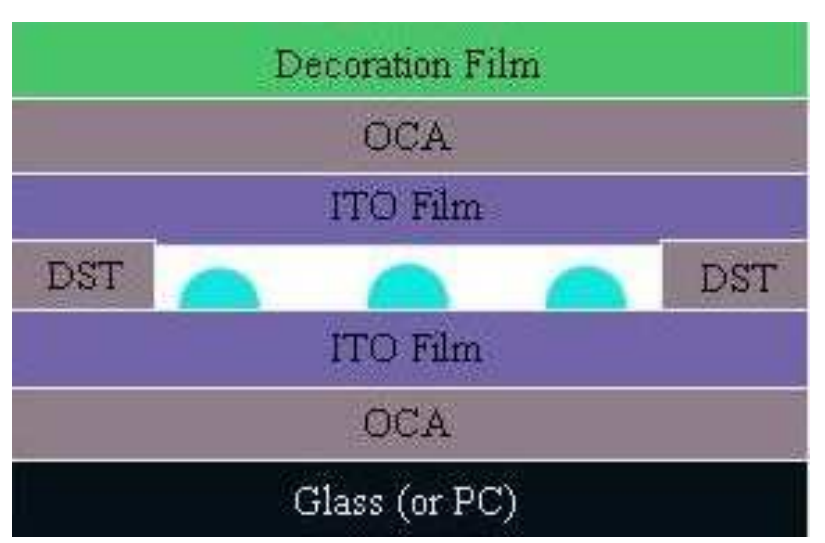

Fig. 1: The diagram of touch panel structure.

coating target is $\mathrm{Cr} 2 \mathrm{O} 3$. An intelligent coating decision mechanism based on neural network technique is expected to be developed finally.

\section{Neural Network Model}

The four-layered forward neural network is mainly taken as the estimation model which is shown in Fig. 2. It consists of one input layer, two hidden layers and one output layer. Each layer is composed of several parallel nodes. The sigmoid function is used as the node's activation function. The error back-propagation (BP) learning algorithm is adopted for network's training. The major steps of BP algorithm could be summarized as follows $[5,8,11,12]$.

Step 1: All weights ( $\left.\omega_{i j} \mathrm{~s}\right)$ are initialized by small random values.

\section{Step 2: Present the input/output pattern.}

Step 3: Calculate the output by using the present value of $\omega_{i j} \mathrm{~s}$.

Step 4: Find the error term, $\delta_{j}$, for all nodes.

Denote $D_{j}$ and $O_{j}$ to be the desired value and the computed value of $j$ th node in output layer. $H_{j}$ is the computed value of $j$ th hidden node. The error terms for all nodes can be calculated as follows.

For the output node $j$

$$
\delta_{j}=\left(D_{j}-O_{j}\right) O_{j}\left(1-O_{j}\right)
$$

For the hidden node $j$

$$
\delta_{j}=H_{j}\left(1-H_{j}\right) \sum_{k} \omega_{j k} \delta_{k}
$$

where $k$ is over all nodes in the layer above node $j$.

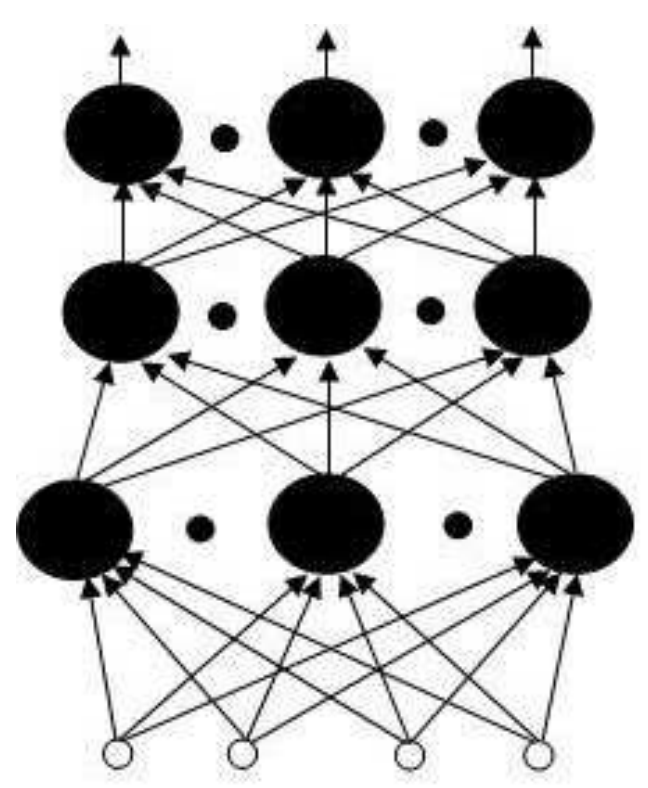

Fig. 2: The diagram of a four-layered feed-forward neural network.

Step 5: All weights can be updated by

$$
\omega_{i j}(n+1)=\omega_{i j}(n)+\alpha \delta_{j} X_{i}+\zeta\left(\omega_{i j}(n)-\omega_{i j}(n-1)\right)
$$

where $X_{i}$ is the ith input signal of node $j . \alpha$ is the learning rate and $\zeta$ is the momentum.

Step 6: Present another input/output pattern and go back to Step 2.

\section{Simulations}

The evaporation process of film coating is an important work for TP manufacturing. Many control parameters and influencing factors need to be considered before the process is started. That is the reason why this work is usually taken by the engineer who has the full experience in this area. Unfortunately, trial-and-error is still the common method taken by lots of manufacturing companies. Thus, if an AI decision system for the manufacturing control parameters setting could be developed, this system certainly can help the engineer to accomplish the evaporation work easily even the engineer lacks the experience.

In this research, the data of decoration film provided by an optical company are analyzed and simulated. The example of data for study is presented in Table 1. Except the TP transmittance, the possible influencing factors are collected. They are the value of quartz crystal deposition monitor, the holder's rotation speed, the placed position of film, the thickness of coating target $\mathrm{Cr}$ and the 
thickness of coating target $\mathrm{Cr}_{2} \mathrm{O}_{3}$. The research aims to catch the complicated relationship between TP film?s transmittance and its all possible influencing factors by neural network automatically $[5,6]$. It is well known that BP algorithm usually has the problem of local minimum learning. Thus, in order to fairly evidence the feasibility of neural network, Set-A, Set-B, Set-C and Set-D, four different data sets randomly re-organized from the original data were simulated by neural model respectively. For each data set, the number of data used for network's training is 100 and the number of data used for network's test is 44. The mean absolute error (MAE) and the mean absolute percentage error (MAPE) are the judging criteria used for the network's performance.

The size of m-2-2-1 neural model is used in our studies, where $\mathrm{m}$ is the number of input nodes. Several simulations were carried out by using different inputs in order that the best input combination could be found. From the simulation results shown, we conclude that the rotation speed of holder, $\mathrm{Cr}$ thickness and $\mathrm{Cr}_{2} \mathrm{O}_{3}$ thickness are three most important influencing factors in the coating process. They have the higher correlations with the transmittance. Tables 2 to 9 list the statistics performed by different sizes of neural model. These statistic results clearly show that if the proper influencing factors could be determined, the neural network technique indeed is a good and suitable tool to do the estimation work for TP film's optical property. In other words, the accurate transmittance of decoration film is possibly to be estimated and controlled before the coating process is started.

\section{Conclusion}

This research presents the transmittance estimations of TP decoration film by using neural network with BP learning algorithm. From the results shown in Table 2, Table 3, Table 5 and Table 8, the estimation accuracy performed by neural network is quite good, if three influencing factors, the rotation speed of holder, $\mathrm{Cr}$ thickness and $\mathrm{Cr}_{2} \mathrm{O}_{3}$ thickness, could be included into the inputs of network's training. The best average MAPE is about $1.89 \%$. This research results evidence that the neural network is a suitable technique used for catching the complicated relationship between the optical property of TP coating film and the relevant parameters of coating process. In other words, an AI decision system used for controlling TP evaporation process is possibly developed. Undoubtedly, such a smart decision system could make several advantages for the optical company, like helping the junior engineer to produce the qualified TP film, avoiding the trial-and-error step, saving the working time and saving the company cost.
Table 1: The example of simulation data.

\begin{tabular}{|c|c|c|c|c|c|}
\hline $\begin{array}{l}\text { Quarts } \\
\text { aystal }\end{array}$ & $\begin{array}{l}\text { Holder's } \\
\text { rotntion } \\
\text { speed }\end{array}$ & $\begin{array}{l}\text { Substrate } \\
\text { position }\end{array}$ & $\underset{\text { thichness }}{\mathrm{Cr}}$ & $\begin{array}{c}\text { Cr } 0 \\
\text { thichare }\end{array}$ & $\begin{array}{c}\text { Transm ttarce } \\
(\mathrm{T} \% / \dot{ })\end{array}$ \\
\hline 625 & 10 & 6 & 50 & 30 & 59 \\
\hline 632 & 15 & 11 & 100 & $\infty 0$ & 32 \\
\hline 631 & 10 & 6 & 150 & 130 & 25 \\
\hline 631 & 10 & 5 & 150 & 130 & 23 \\
\hline 632 & 15 & 8 & 100 & 80 & 31 \\
\hline 632 & 15 & 9 & 100 & 80 & 32 \\
\hline 621 & 5 & 1 & 100 & 80 & 37 \\
\hline 632 & 20 & 5 & 150 & 80 & 30 \\
\hline 633 & 15 & 7 & 100 & 80 & 33 \\
\hline 632 & 10 & 6 & 150 & 130 & 22 \\
\hline 631 & 15 & 5 & 100 & 80 & 31 \\
\hline 630 & 20 & 3 & 100 & $\infty 0$ & 36 \\
\hline 621 & 20 & 2 & 50 & 30 & 61 \\
\hline 621 & 15 & 1 & 50 & 30 & 61 \\
\hline 623 & 5 & 11 & 50 & 30 & 58 \\
\hline 621 & 20 & 8 & 50 & 30 & 61 \\
\hline \begin{tabular}{|l}
623 \\
\end{tabular} & 5 & 12 & 50 & 30 & 58 \\
\hline 631 & 10 & 4 & 150 & 130 & 28 \\
\hline 631 & 10 & 11 & 150 & 130 & 23 \\
\hline 621 & 20 & 12 & 50 & 30 & 61 \\
\hline 630 & 20 & 12 & 100 & 80 & 37 \\
\hline$\therefore$ & $:$ & $:$ & : & $:$ & $:$ \\
\hline$\therefore$ & $\therefore$ & 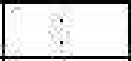 & $\therefore$ & $\therefore$ & : \\
\hline$\therefore$ & $\because$ & $\vdots$ & $:$ & $:$ & : \\
\hline$:$ & $\because$ & $:$ & : & $\therefore$ & $:$ \\
\hline
\end{tabular}

\section{Acknowledgement}

This work was supported by the National Science Council of Republic of China under contract No. NSC-99-2221-E214-001 
Table 2: The statistics performed by five-input NN model.

Inputs: quartz crystal value, holder's rotation speed, substrate position of pane1, Cr thickness, $\mathrm{Cr}_{2} \mathrm{O}_{3}$ thickness

\begin{tabular}{|c|c|c|c|c|}
\hline \multirow{2}{*}{ Data } & \multicolumn{2}{|c|}{ MAE } & \multicolumn{2}{c|}{ MAPE (\%) } \\
\cline { 2 - 5 } & Training & Test & Training & Test \\
\hline Set-A & 0.5740 & 0.7273 & 1.6544 & 2.0347 \\
\hline Set-B & 0.6234 & 0.7452 & 1.7478 & 2.1748 \\
\hline Set-C & 0.6205 & 0.6944 & 1.7551 & 1.9895 \\
\hline Set-D & 0.6449 & 0.7216 & 1.9061 & 1.8085 \\
\hline Avg. & $\mathbf{0 . 6 1 5 7}$ & $\mathbf{0 . 7 2 2 1}$ & $\mathbf{1 . 7 6 5 8}$ & $\mathbf{2 . 0 0 1 9}$ \\
\hline
\end{tabular}

Table 3: The statistics performed by $1^{\text {st }}$ four-input NN model.

\begin{tabular}{|c|c|c|c|c|}
\hline \multicolumn{5}{|c|}{$\begin{array}{l}\text { Inputs: holder's rotation speed, substrate position } \\
\text { of panel, } \mathrm{Cr} \text { thickness, } \mathrm{Cr}_{2} \mathrm{O}_{3} \text { thickness }\end{array}$} \\
\hline \multirow{2}{*}{ Data } & \multicolumn{2}{|c|}{ MAE } & \multicolumn{2}{|c|}{ MAPE $(\%)$} \\
\hline & Training & Test & Training & Test \\
\hline Set-A & 0.5541 & 0.6902 & 1.5843 & 1.8693 \\
\hline Set-B & 0.6048 & 0.7326 & 1.6674 & 2.1034 \\
\hline Set-C & 0.6186 & 0.6574 & 1.7220 & 1.8575 \\
\hline Set-D & 0.6355 & 0.7086 & 1.8777 & 1.7541 \\
\hline Avg. & 0.6033 & 0.6972 & 1.7129 & 1.8961 \\
\hline
\end{tabular}

Table 4: The statistics performed by $2^{\text {nd }}$ four-input NN model. Inputs: quartz crystal value, substrate position of panel, $\mathrm{Cr}$ thickness, $\mathrm{Cr}_{2} \mathrm{O}_{3}$ thickness

\begin{tabular}{|c|c|c|c|c|}
\hline \multirow{2}{*}{ Data } & \multicolumn{2}{|c|}{ MAE } & \multicolumn{2}{c|}{ MAPE (\%) } \\
\cline { 2 - 5 } & Training & Test & Training & Test \\
\hline Set-A & 0.8531 & 0.8422 & 2.7020 & 2.5272 \\
\hline Set-B & 0.8550 & 0.9466 & 2.6882 & 2.9071 \\
\hline Set-C & 0.8251 & 0.9677 & 2.5853 & 2.9699 \\
\hline Set-D & 0.8973 & 0.8943 & 2.8886 & 2.5034 \\
\hline Avg. & $\mathbf{0 . 8 5 7 6}$ & $\mathbf{0 . 9 1 2 7}$ & $\mathbf{2 . 7 1 6 0}$ & $\mathbf{2 . 7 2 6 9}$ \\
\hline
\end{tabular}

Table 5: The statistics performed by $3^{r d}$ four-input NN model.

\begin{tabular}{|c|c|c|c|c|}
\hline \multicolumn{5}{|c|}{$\begin{array}{l}\text { Inputs: quartz crystal value, holder's rotation } \\
\text { speed, Cr thickness, } \mathrm{Cr}_{2} \mathrm{O}_{3} \text { thickness }\end{array}$} \\
\hline \multirow{2}{*}{ Data } & \multicolumn{2}{|c|}{ MAE } & \multicolumn{2}{|c|}{ MAPE $(\%)$} \\
\hline & Training & Test & Training & Test \\
\hline Set-A & 0.6956 & 0.6276 & 2.0196 & 1.7338 \\
\hline Set-B & 0.6566 & 0.6751 & 1.8639 & 1.8957 \\
\hline Set-C & 0.6523 & 0.7024 & 1.8317 & 2.0148 \\
\hline Set-D & 0.6525 & 0.7942 & 1.9398 & 2.0391 \\
\hline Avg. & 0.6643 & 0.6998 & 1.9137 & 1.9208 \\
\hline
\end{tabular}

Table 6: The statistics performed by $1^{\text {st }}$ three-input NN model. Inputs: quartz crystal value, $\mathrm{Cr}$ thickness, $\mathrm{Cr}_{2} \mathrm{O}_{3}$ thickness

\begin{tabular}{|c|c|c|c|c|}
\hline \multirow{2}{*}{ Data } & \multicolumn{2}{|c|}{ MAE } & \multicolumn{2}{c|}{ MAPE (\%) } \\
\cline { 2 - 5 } & Training & Test & Training & Test \\
\hline Set-A & 0.9573 & 0.8401 & 3.0257 & 2.5033 \\
\hline Set-B & 0.9137 & 0.8929 & 2.8905 & 2.7556 \\
\hline Set-C & 0.8991 & 1.0101 & 2.7804 & 3.1371 \\
\hline Set-D & 0.9251 & 0.8995 & 3.0015 & 2.5032 \\
\hline Avg. & $\mathbf{0 . 9 2 3 8}$ & $\mathbf{0 . 9 1 0 6}$ & $\mathbf{2 . 9 2 4 5}$ & $\mathbf{2 . 7 2 4 8}$ \\
\hline
\end{tabular}

Table 7: The statistics performed by $2^{\text {nd }}$ three-input NN model.

\begin{tabular}{|c|c|c|c|c|}
\hline \multicolumn{5}{|c|}{$\begin{array}{l}\text { Inputs: substrate position of panel, } \mathrm{Cr} \text { thickness, } \\
\mathrm{Cr}_{2} \mathrm{O}_{3} \text { thickness }\end{array}$} \\
\hline \multirow{2}{*}{ Data } & \multicolumn{2}{|c|}{ MAE } & \multicolumn{2}{|c|}{ MAPE (\%) } \\
\hline & Training & Test & Training & Test \\
\hline Set-A & 1.8423 & 1.9126 & 5.5174 & 5.3602 \\
\hline Set-B & 1.8157 & 1.7833 & 5.4165 & 5.1642 \\
\hline Set-C & 1.7517 & 2.0462 & 5.1468 & 6.3702 \\
\hline Set-D & 1.8862 & 1.7725 & 5.6685 & 4.8626 \\
\hline Avg. & 1.8240 & 1.8787 & 5.4373 & 5.4393 \\
\hline
\end{tabular}


Table 8: The statistics performed by $3^{\text {rd }}$ three-input NN model.

\begin{tabular}{|c|c|c|c|c|}
\hline \multicolumn{5}{|c|}{$\begin{array}{l}\text { Inputs: holder's rotation speed, } \mathrm{Cr} \text { thickness, } \mathrm{Cr}_{2} \mathrm{O}_{3} \\
\text { thickness }\end{array}$} \\
\hline \multirow{2}{*}{ Data } & \multicolumn{2}{|c|}{ MAE } & \multicolumn{2}{|c|}{ MAPE $(\%)$} \\
\hline & Training & Test & Training & Test \\
\hline Set-A & 0.6503 & 0.6157 & 1.8728 & 1.7119 \\
\hline Set-B & 0.6581 & 0.6823 & 1.8671 & 1.9128 \\
\hline Set-C & 0.6518 & 0.7146 & 1.8300 & 2.0546 \\
\hline Set-D & 0.6474 & 0.7456 & 1.9299 & 1.8992 \\
\hline Avg. & 0.6519 & 0.6896 & 1.8750 & 1.8946 \\
\hline
\end{tabular}

Table 9: The statistics performed by two-input NN model.

\begin{tabular}{|c|c|c|c|c|}
\hline \multirow{2}{*}{ Inputs: $\mathrm{Cr}$ thickness and $\mathrm{Cr}_{2} \mathrm{O}_{3}$ thickness } \\
\hline \multirow{2}{*}{ Data } & \multicolumn{2}{|c|}{ MAE } & \multicolumn{2}{c|}{ MAPE (\%) } \\
\cline { 2 - 5 } & Training & Test & Training & Test \\
\hline Set-A & 1.8731 & 1.8723 & 5.5752 & 5.2641 \\
\hline Set-B & 1.8676 & 1.7859 & 5.5442 & 5.1305 \\
\hline Set-C & 1.7718 & 2.0448 & 5.2164 & 6.3065 \\
\hline Set-D & 1.8946 & 1.7356 & 5.7019 & 4.7600 \\
\hline Avg. & $\mathbf{1 . 8 5 1 8}$ & $\mathbf{1 . 8 5 9 7}$ & $\mathbf{5 . 5 0 9 4}$ & $\mathbf{5 . 3 6 5 3}$ \\
\hline
\end{tabular}

\section{References}

[1] N. L. Dmitruk, A. V. Korovin, Thin Solid Films, 484, 382-388 (2005).

[2] Y. Wu, X. Weng, L. Deng, Journal of Vacuum Science and Technology, 26, 372-376 (2006).

[3] Q. Chen, M. Dfrise, F. Deconinck, IEEE Transactions on Pattern Analysis and Machine Intelligence, 16, 1156-1168 (1994).

[4] K. Wang, H. Z. Jia, G. Z. Xia, Spectroscopy and Spectral Analysis, 28, 2713-2716 (2008).

[5] D. J. Huang, F. T. Liu, S. J. Chuang, Y. J. Chen, S. M. T. Wang, R. C. Hwang, The First International Conference on Pervasive Computing, Signal Processing and Applications, 940-943 (2010).

[6] S. M. T. Wang, D. J. Huang, J. P. Yang, Y. J. Chen, H. C. Huang, R. C. Hwang, 2011 IEEE International Conference on Signal Processing, Communications and Computing (ICSPCC 2011), 1080-1083 ( 2011).

[7] G. Z. He, C. X. Xiong, X. L. Yao, Proceedings of SPIE - The International Society for Optical Engineering,
Nanophotonics, Nanostructure, and Nanometrology, II, 683111 (2008).

[8] A. Khotanzad, R. C. Hwang, A. Abaye, D. Maratukulam, IEEE Transactions on Power Systems, 10, 1716-1722 (1995).

[9] B. Zhang, M. Fu, H. Yan, M. A. Jabri, IEEE Transactions on Neural Networks, 10, 939-945 (1999).

[10] C. Wohler, J. K. Anlauf, IEEE Transactions on Neural Networks, 10, 1531-1536 (1999).

[11] H. C. Huang, R. C. Hwang, and J. G. Hsieh, International Journal of Electrical Power and Energy Systems, 24, 245-250 (2002).

[12] Y. J. Chen, T. C. Huang, and R. C. Hwang, Information Sciences, 167, 77-86 (2004). 


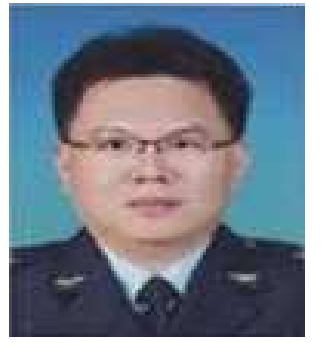

Du-Jou Huang received his MS degrees in Electrical Engineering from I-Shou University in year 2004. Currently, he is pursuing his Ph.D. degree at I-Shou University. His research interests are in the areas of neural network and its applications.

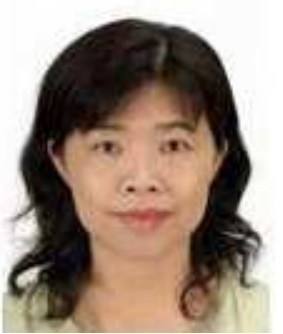

and conferences.

Yu-Ju Chen currently is an assistant professor of Information Management Department at Cheng Shu University. Her research interests include the areas of artificial intelligence, fuzzy theory and signal processing. She has published more than 60 papers in various journals

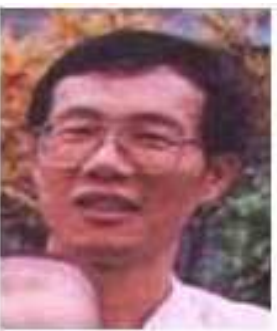

Chuo-Yean received his $\mathrm{PhD}$ degree in Electrical Engineering from I-Shou University, Taiwan, ROC. Currently he is an associate professor of Electrical Engineering Department at Cheng Shu University. His research interests include the areas of artificial intelligence, fuzzy theory and power systems.

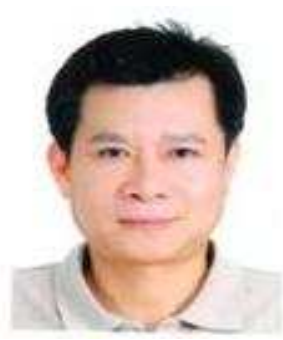

Huang-Chu Huang received his Ph.D. degree in Electrical Engineering from National Sun Yat-Sen University, Taiwan, in year 2001. Currently, he is an associate professor and the chair of Electronic Communication Department, National Kaohsiung Marine University, Kaohsiung City, Taiwan, ROC. His research interests are in the areas of control, power signal prediction and neural network applications.

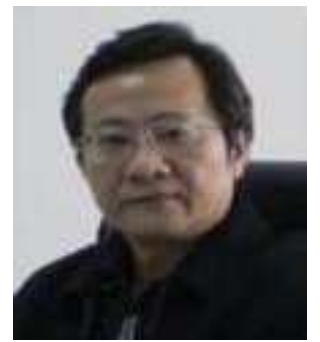

Rey-Chue Hwang received his $\mathrm{PhD}$ degree in Electrical Engineering from Southern Methodist University, Dallas, TX, in 1993. Currently, he is a full professor of Electrical Engineering Department, I-Shou University, Taiwan, ROC. Dr. Hwang has published more than 180 papers in various journals and conferences in the areas of artificial intelligent system, signal processing and fuzzy control. He is now a Fellow of IET and a senior member of IEEE. He chartered the IEEE CIS Chapter, Tainan Section and served as the co-chair and chair from year 2004 to year 2009 . 